\title{
Creatividad y legado: adornos corporales en el sitio arqueológico Nejapa, Nicaragua
}

Creativity and legacy: body adornments at the archaeological site of Nejapa, Nicaragua

Kevin González Hodgson

Centro Arqueológico de Documentación e Investigación CADI-UNAN-Managua.

orcid: https://orcid.org/0000-0003-1127-4309

Correo electrónico: hodgsonk27@hotmail.com
Recibido: 07-10-2018

Aceptado: 25-08-2018

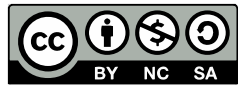

\section{Resumen}

En este estudio se analiza los elementos corporales recuperados durante la excavación estratigráfica realizado en dos contextos domésticos en el sitio arqueológico Nejapa, en el departamento de Managua recurriendo para ello a técnicas de análisis métricos y morfológicos con el objetivo de lograr una caracterización detallada de la variabilidad de diseños recuperados en el registro arqueológico. Los resultados obtenidos sostienen que los adornos corporales simbolizan un lenguaje no verbal que transmiten información esencial de nuestra naturaleza social y cultural por lo que eventualmente, están vinculados con la tradición y la identidad de la población local asentado en esta zona de la capital nicaragüense.

Palabras clave: Adornos corporales, aproximación interpretativa, cultura material, identidad, Nejapa Nicaragua.

\begin{abstract}
This study analyzes the body items recovered during the stratigraphic excavation in two contexts domestic archaeological Nejapa, in the Department of Managua by using this metric analysis techniques and morphological aiming to achieve a detailed characterization of the variability of designs in the archaeological record. The results hold the body adornments to symbolize a non-verbal language that convey information essential to our social and cultural nature are eventually linked to tradition and the identity of the local population settled in this area of the Nicaraguan capital.
\end{abstract}

Keywords: Body adornments, interpretative approach, material culture, identity, Nejapa Nicaragua. 


\section{Introducción}

Este artículo tiene por objetivo central analizar los adornos corporales encontrados en la excavación estratigráfica efectuado en dos contextos domésticos correspondientes al sitio arqueológico Nejapa1 en el departamento de Managua y vinculado al período cultural (800 d.C.-1550 d.C.); y de manera más detallada el estudio implicó por un lado una caracterización tecno-morfológica e identificación del tipo y variedad de materias primas y por otro determinar asociaciones entre las variables señaladas que resultaron de suma importancia en la elaboración de los adornos. Para ello, se examinó 109 piezas completas y fragmentadas de una muestra original de 164 ejemplares lavados y clasificados por el Br. Emilio Carranza y presentado como ensayo en el XXVII Jornada Universitaria de Desarrollo Científico del departamento de Historia en el año 2008.

En este caso su servidor retoma dichos materiales a raíz de ampliar el conocimiento en torno no sólo a cuentas de collar sino al conjunto ornamental seleccionado ahora (100 cuentas de diversas tipologías, 2 colgantes una de piedra y otra cerámica como también, 7 extensores ligados a narigueras $\mathrm{u}$ orejeras) que a partir, de sus características morfológicas y morfométricas permitieron discutir sobre el rol de los mismos en la construcción de la identidad de sus creadores por cuanto agrega una cualidad expresa al cuerpo humano que desde tiempos inmemorables el ser humano comenzó a adornar su entorno incluyendo su propia persona. La existencia de este tipo de evidencia a nivel nacional ha sido reportada esencialmente en contextos funerarios regularmente junto a otros elementos ofrecidos cerámica, piedra y huesos que reflejan relaciones sociales diarias en el pasado y que también son expresiones simbólicas de estatus, siendo de esta forma el tema de la cultura material la base de este estudio y la que permite la construcción de interpretaciones preliminares. A los efectos, de este estudio retomamos el concepto de adornos corporales como constructo de identidad por lo que partimos de la idea de que estos elementos son artículos de uso personal que refieren directamente al individuo y que su práctica en el pasado convierte a las personas en portadoras de mensajes simbólicos varios lo que supone la incorporación de elementos agentivos propios de alteridad $u$ otredad en la constitución de las personas.

\section{Nejapa y el contexto de objetos ornamentales}

Las primeras exploraciones de este sitio fueron en el año 2006 con un reconocimiento superficial del sitio y desde entonces consecutivamente durante cinco temporadas (2007-2012) se excavó en los montículos 1 (M-1) y 5 (M5) con la participación tanto de estudiantes y docentes del Centro Arqueológico de Documentación e Investigación (CADI) de la UNAN-Managua; la excavación determinó aue las estructuras estudiadas corresponden a

\section{Mapa 1, 2 y 3. Localización del sitio arqueológico; cartografía del sitio; topografía y curvas a nivel. Cortesía: CADI-UNAN-Managua/Alcaldía de Managua.}

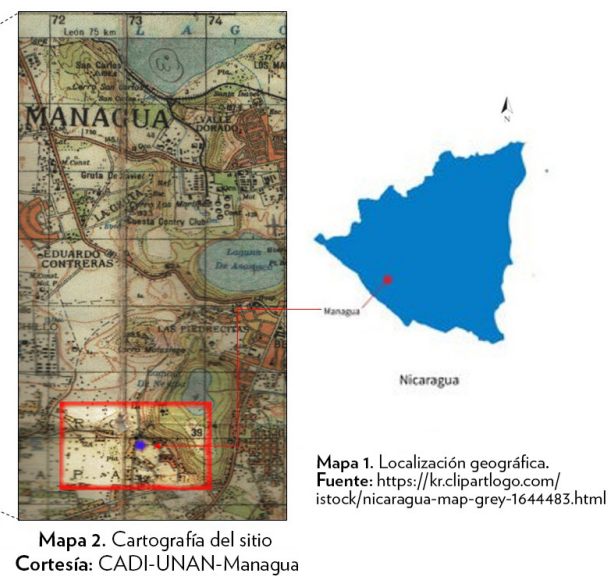

unidades habitacionales (viviendas indígenas) que se caracterizaron por la constante aparición de un alto índice de material cerámico (bordes, cuerpos, cuellos, asas, pesas de red), lítica, restos paleobotánicos, además de elementos propios del sistema constructivo (muros de contención en formas de lajas), agujeros de poste (horcón), áreas de combustión y la relevancia de un significativo número de adornos corporales que 


\begin{tabular}{|c|c|c|c|c|c|c|c|c|c|c|}
\hline \multirow{3}{*}{$\begin{array}{l}\text { Elemento } \\
\text { Corporal }\end{array}$} & \multirow{3}{*}{$\begin{array}{c}\text { Materia } \\
\text { prima }\end{array}$} & \multicolumn{2}{|c|}{ Dimensiones en $\mathrm{cm} / \mathrm{mm}$} & \multicolumn{3}{|c|}{ Manufactura } & \multirow{2}{*}{\multicolumn{3}{|c|}{ Decoración }} & \multirow[t]{3}{*}{ Descripciones } \\
\hline & & \multirow{2}{*}{ Largo } & \multirow{2}{*}{ Ancho } & \multirow[b]{2}{*}{$\begin{array}{l}\text { Técnica de } \\
\text { elaboración }\end{array}$} & \multicolumn{2}{|c|}{ Tipo de perforación } & & & & \\
\hline & & & & & $\begin{array}{l}\text { Forma Corte } \\
\text { del Orificio }\end{array}$ & $\begin{array}{l}\text { Forma del } \\
\text { Contorno }\end{array}$ & $\begin{array}{l}\text { Pintura/ } \\
\text { Engobe }\end{array}$ & $\begin{array}{l}\text { Incisión/ } \\
\text { impresión }\end{array}$ & Esculpido & \\
\hline \multicolumn{11}{|c|}{ Cuentas triangulares } \\
\hline $\begin{array}{c}\text { Cuenta } \\
\text { Triangular }\end{array}$ & Cerámica & $0.9 \mathrm{~mm}$ & $0.5 \mathrm{~mm}$ & $\begin{array}{l}\text { Perforación } \\
\text { (para dar } \\
\text { forma) }\end{array}$ & Cónico & Circular & Marrón & $\begin{array}{l}\text { Impresión en } \\
3 \text { areas }\end{array}$ & Esculpido & $\begin{array}{l}\text { Forma triangular con } \\
\text { impresiones que defin } \\
\text { en una especie de ojo. }\end{array}$ \\
\hline \multicolumn{11}{|c|}{ Cuentas globulares } \\
\hline $\begin{array}{l}\text { Fragmento } \\
\text { cuenta globular }\end{array}$ & Cerámica & $1 \mathrm{~cm}$ & $0.5 \mathrm{~mm}$ & Pulido & Cónico & Circular & Marrón & ---- & ---- & $\begin{array}{l}\text { Morfológicamente la pi } \\
\text { eza tiene similitud con } \\
\text { los botones. }\end{array}$ \\
\hline \multicolumn{11}{|c|}{ Cuentas cilíndricas } \\
\hline $\begin{array}{l}\text { Cuenta } \\
\text { cilíndrica }\end{array}$ & Cerámica & $1 \mathrm{~cm}$ & $0.5 \mathrm{~mm}$ & $\begin{array}{l}\text { Perforación } \\
\text { (para dar } \\
\text { forma) }\end{array}$ & $\begin{array}{l}\text { Cilíndrica } \\
\text { (tubular) }\end{array}$ & Circular & $\begin{array}{l}\text { Engobe } \\
\text { Negro }\end{array}$ & $\begin{array}{|lr|}\begin{array}{l}\text { Incisión } \\
\text { forma }\end{array} & \text { en } \\
\text { cular } & \text { cir- } \\
\end{array}$ & Esculpido & $\begin{array}{l}\text { Cuenta de dos partes, } \\
\text { tiene } 6 \text { incrustaciones } \\
\text { (especie de ojo). }\end{array}$ \\
\hline \multicolumn{11}{|c|}{ Cuentas discoidales } \\
\hline $\begin{array}{l}\text { Fragmento } \\
\text { cuenta } \\
\text { discoidal }\end{array}$ & Cerámica & $0.2 \mathrm{~mm}$ & iámetro & $\begin{array}{l}\text { Perforación } \\
\text { (para dar } \\
\text { forma) y } \\
\text { pulido }\end{array}$ & $\begin{array}{l}\text { Cónico } \\
\text { (perforado } \\
\text { central) }\end{array}$ & Circular & Marrón & $-\cdots--$ & ---- & $\begin{array}{l}\text { Forma de disco (diámet } \\
\text { ro mínimo a } 0.2 \mathrm{~mm} \text { ), } \\
\text { con caras circulares } \\
\text { sección plana, con per- } \\
\text { foración central }\end{array}$ \\
\hline $\begin{array}{l}\text { Cuenta } \\
\text { discoidal }\end{array}$ & Piedra & $0.4 \mathrm{de}$ & metro & $\begin{array}{l}\text { Perforación y } \\
\text { pulido }\end{array}$ & $\begin{array}{c}\text { Cónico } \\
\text { (perforado } \\
\text { central) }\end{array}$ & Circular & Verde & ---- & $-\cdots$ & $\begin{array}{l}\text { Materia prima piedra } \\
\text { verde (especie de ám } \\
\text { bar, jade o jadeíta) }\end{array}$ \\
\hline \multicolumn{11}{|c|}{ Cuentas esféricas } \\
\hline $\begin{array}{l}\text { Cuenta esférica } \\
\text { pequeña }\end{array}$ & Cerámica & $0.6 \mathrm{~mm}$ & iámetro & $\begin{array}{l}\text { Patillaje } \\
\text { (técnica de } \\
\text { Churro) }\end{array}$ & Cónico & Circular & Marrón & $-\cdots--$ & ---- & $\begin{array}{l}\text { Se observa la técnica de } \\
\text { churro }\end{array}$ \\
\hline \multicolumn{11}{|c|}{ Cuentas irregulares } \\
\hline $\begin{array}{l}\text { Cuenta } \\
\text { irregular }\end{array}$ & Cerámica & $0.6 \mathrm{~mm}$ & $0.4 \mathrm{~mm}$ & $\begin{array}{l}\text { Perforación } \\
\text { (para dar } \\
\text { forma) }\end{array}$ & Cónico & Circular & Marrón & $-\cdots$ & $-\cdots$ & ---- \\
\hline \multicolumn{11}{|c|}{ Colgante sobre piedra } \\
\hline $\begin{array}{l}\text { Colgante sobre } \\
\text { piedra }\end{array}$ & Piedra & $1.7 \mathrm{~cm}$ & $1.3 \mathrm{~cm}$ & $\begin{array}{l}\text { Perforación } \\
\text { (para dar } \\
\text { forma) }\end{array}$ & ----- & ---- & $\begin{array}{l}\text { Gris/ } \\
\text { negro }\end{array}$ & $\begin{array}{l}\text { Incisión } \\
\text { vertical }\end{array}$ & ---- & $\begin{array}{l}\text { Piedra dura de mediano } \\
\text { tamaño y línea incisa de } \\
\text { manera vertical en am } \\
\text { bas caras }\end{array}$ \\
\hline \multicolumn{11}{|c|}{ Colgante sobre cerámica } \\
\hline $\begin{array}{l}\text { Colgante sobre } \\
\text { cerámica }\end{array}$ & Cerámica & $1 \mathrm{~cm}$ & $0.6 \mathrm{~mm}$ & $\begin{array}{l}\text { Perforación } \\
\text { (para dar for- } \\
\text { ma) y pulido }\end{array}$ & Bicónico & Semi-circular & Marrón & $-\cdots$ & $\cdots-$ & $\begin{array}{l}\text { La forma es un triángulo } \\
\text { irregular }\end{array}$ \\
\hline \multicolumn{11}{|c|}{ Extensores (nariguera/orejeras) } \\
\hline $\begin{array}{l}\text { Fragmento nar- } \\
\text { iguera/oreja }\end{array}$ & Cerámica & $0.9 \mathrm{~mm}$ & $0.7 \mathrm{~mm}$ & $\begin{array}{l}\text { Perforación } \\
\text { (para dar for- } \\
\text { ma) y pulido }\end{array}$ & Cónico & Circular & Negro & $\begin{array}{l}\text { Incisión } \\
\text { Diagonal }\end{array}$ & $\cdots--$ & $\begin{array}{l}3 \text { fragmentos remontan e } \\
\text { objeto en sí }\end{array}$ \\
\hline
\end{tabular}

Tabla 1. Síntesis de las características morfológicas y métricas de elementos corporales del sitio arqueológico Nejapa

permitieron entender aspectos vinculados con la forma de vida de los antiguos habitantes en Nejapa. El territorio en estudio comprende la parte sur-occidental de la ciudad de Managua (mapas 1, 2 y 3) correspondiente al distrito III y conformado por una serie de elementos geográficos preponderantes como la Laguna de Nejapa (al Noroeste) de origen volcánico y otras fuentes hídricas relativamente cercanas entre ellos la Laguna de Asososca $(2 \mathrm{~km})$ y el Lago Cocibolca $(5-6 \mathrm{~km})$ aproximadamente; además, en el área de influencia del sitio se encuentra el cerro Motastepe (al Norte) que hacen que la zona se caracterice por tener suelos fértiles precisamente por los depósitos de sedimentos volcánicos, clima de sabana tropical de prolongación seco con vegetación variado y biodiversidad considerable. Doce estructuras monticulares o casas indígenas de dimensiones variadas en aproximadamente 9 manzanas de terreno evidencian el desarrollo de un amplio asentamiento prehispánico situado propiamente en el kilometro 9 1/2 de la carretera vieja que conduce al departamento de León en el sector de Villa Fátima. 


\section{Materiales y metodología}

Se hizo un levantamiento del material depositado en el laboratorio del CADI referido a 7 campañas de excavación en su mayoría de las unidades estratigráficas $(\mathrm{UE}-3)^{2}$. Además, se clasificaron los rasgos estéticos combinando técnicas de análisis métricos en $(\mathrm{cm} /$ $\mathrm{mm}$ ), morfológicos ${ }^{3} \mathrm{y}$ análisis arqueológico o caracterización simbólica del adorno personal como parte de la indumentaria utilizado. En la limpieza de la muestra se hizo uso de alcohol al100\% o agua, frotando con cuidado que no dañara los objetos. También, se fotografiaron, dibujaron y examinaron con lámparas fluorescente de lupa integrada que registró los atributos de los ejemplares seleccionados.

\section{Resultados y discusión}

A continuación, se describe la información procedente del acervo portable de los adornos corporales analizados, por tal razón en el proceso de clasificación de los materiales se consideraron seis esferas de análisis: elemento, materias primas, dimensiones, técnica de manufactura y tipo de perforación, decoración y estado de conservación de los objetos.

Los resultados permitieron determinar tres grupos de materiales que se describen a partir, de las características morfológicas y estéticas entre ellas cuentas de collar de tipología triangular (1), globular (34), cilíndrica (20), cuenta discoidal (33) definido así, por asemejarse a pequeños discos, cuenta esférica (11), cuenta irregular (1), al igual que (1)

colgante sobre piedra, (1) colgante sobre cerámica y (7) extensores que eventualmente corresponden a narigueras/orejeras o asociados a éste; para la elaboración de este tipo de objetos se utilizaron materias primas como el barro, y la piedra verde escasamente identificado apenas una cuenta de collar; Ante la ausencia de ese tipo de materia prima es oportuno plantear la hipótesis sobre una ausencia casi total del mismo en Nicaragua entre otras razones porque "hubo una barrera social y política que no facilitó el intercambio de estos bienes entre sociedades del norte de Costa Rica y sus vecinas de Nicaragua del 500 a.n.e., al 700 n.e. y que el intercambio de materia prima y artefactos ocurrió por vía marítima" (Salgado y Guerrero, 2005:53).

Por otra parte, las dimensiones de los objetos analizados igualmente las variables métricas indican rangos diferenciales en cuanto al tamaño de las cuentas por ejemplo, en promedio de longitud ( \pm desde $0.4 \mathrm{~mm}$ hasta alcanzar $1.7 \mathrm{~cm}$ de diámetro), ( \pm en promedio desde $0.2 \mathrm{~mm}$ hasta $2.2 \mathrm{~cm}$ ) para su anchura. Sobre las formas de trabajo o técnicas de manufactura utilizados en este tipo de adorno corporal es bastante difícil determinar con certeza sobre esa variable, lo que sí se sabe es que de acuerdo a su estructura morfológica sugieren la utilización de la técnica de perforación (para dar forma) y pulido utilizado para suavizar las aristas (bordes de las piezas) y el uso de la técnica del pastillaje o técnica de churros, como lo sugiere una muestra analizada y de los cuales se determina la forma de las piezas precisamente, resultado de este último tratamiento externo en la confección de los mismos.

El tipo de extracción en las muestras fue eventualmente utilizando una especie de perforador (posiblemente hueso o madera) para producir el orificio de incrustación de los objetos, que en el caso de las formas del corte del orificio realizado presenta elementos variados según, la técnica empleado la mayor proporción

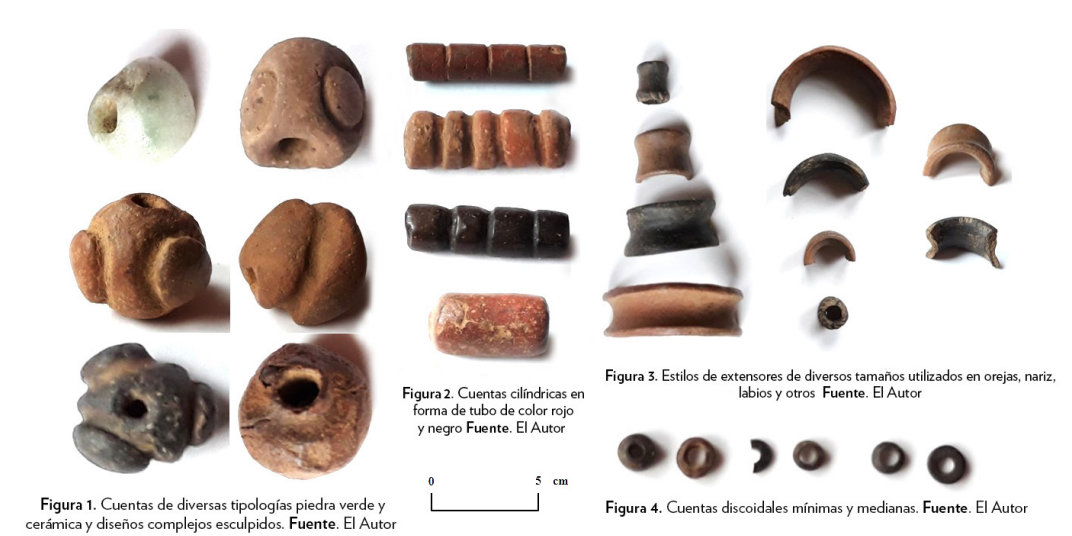


Identificación de cuentas de collar y extensores de manufactura diversa

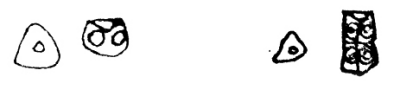

Figuras 5 y 6. Cuentas triangulares de $0.9 \mathrm{~mm} \times 0.5 \mathrm{~mm}$ y $0.6 \mathrm{~mm} \times 0.2 \mathrm{~mm}$

৫๑॰ ๑
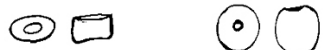

Figuras 7,8 y 9. Cuentas discoidales mínimos y medianos con perforación central y cuentas globulares $0.2 \mathrm{~mm}$,
$0.3 \mathrm{~mm}$ y $0.5 \mathrm{~mm}$

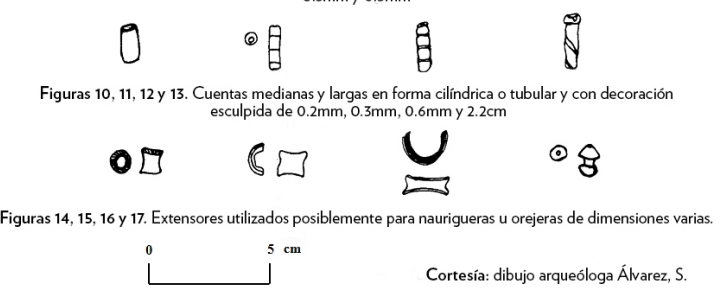

Figuras del 5 al 17. Identificación de collar y extensores de manufactura diversas. Cortesía. Dibujo arqueóloga Álvarez, S.

se resumen hacia formas cónicas (37) y formas cónicas con perforado central (45), pero también se presentan con cortes en forma cilíndrica o tubular (25) y bicónica (2); siempre en el análisis de la perforación específicamente, en la forma del contorno de las cuentas todas (109) tienen contorno circular.

Con respecto, al tratamiento de la superficie de las muestras en ocasiones se lograron observar pigmentos de color marrón. café v negro aue sirvieron de engobe o

\section{Cuentas de collar de orificio cónico, bicónico y tubular}
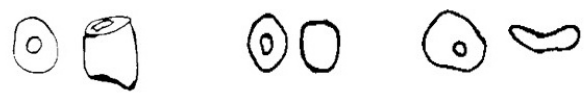

Figuras 18,19 y 20. Cuentas de collar de orificio cónico de $0.9 \mathrm{~mm}, 0.5 \mathrm{~mm}$ y $1 \mathrm{~cm}$
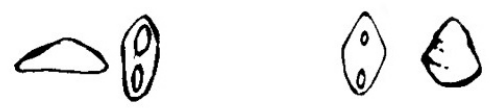

Figuras 21 y 22. Piezas con orificio bicónico de $0.2 \mathrm{~mm}$ y $0.3 \mathrm{~mm}$

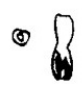

- $\theta$

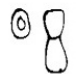

Figuras 23,24 y 25 . Cuentas con orificio tubular de $0.3 \mathrm{~mm}, 0.3 \mathrm{~mm}$ y $0.4 \mathrm{~mm}$

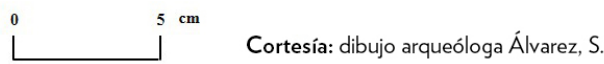

Figuras del 18 al 25. Cuentas de collar de orificio cónico, bicónico y tubular. Cortesía. Dibujo arqueóloga Álvarez, S. simplemente, como pintura al igual que incisiones e impresiones varias; acorde, con el estado de conservación estas se encuentran en buen estado lo que permitió en detalle su análisis científico; no obstante, se reconocen ciertos problemas de deterioro entre ellos erosión o porosidad en su superficie (sobre todo del barro) resultado por una parte de su composición física-química; además, las condiciones medioambientales en las que se dio el descubrimiento generaron procesos de fragmentación (20 objetos partidos) y pérdida del material constituyente en los objetos (posiblemente algún tipo de material plástico, engobe, pintura), que con el tiempo fueron susceptibles de deterioro. Seguidamente, la comparación entre forma, técnica de elaboración, materias primas y las variables métricas (largo y ancho) tiene correlaciones significativas sobre todo en las tipologías de cuentas discoidales mínimas esto según rasgos estéticos y similitud métrica en el orificio realizado en la cuenta.

\section{Función y significados de los adornos}

Una forma adecuada de iniciar la reflexión acerca de los adornos corporales comienza por su definición y desde luego indicar bajo qué términos concebimos este tipo de práctica, en principio son artículos de uso personal diseñadas para ser portadas en el cuerpo humano y su práctica convierte a las personas en portadoras de mensajes socio-culturales, identitarios, simbólicos y estéticos de un grupo específico; por tanto, el primer objeto decorado fue el cuerpo humano en sí, como elemento comunicativo con grupos humanos desde mucho tiempo atrás, que los identificaba en efecto o bien que los diferenciaba de los demás. El significado de este tipo de adorno en culturas ancestrales y presentes tiene diferentes connotaciones desde un significado simbólico vinculado a la espiritualidad o bien de prácticas políticas con el hecho de perforarse o pintarse hasta sencillamente no representar algún significado simplemente estético como pasa hoy. 
A partir, del análisis de los atributos morfológicos en las piezas es posible pensar por ahora que su uso y función estaba relacionado a cuentas, orejeras, narigueras y pendientes como lo sugieren los desgastes en los orificios que permitieron inferir sobre este aspecto y que en el caso de las cuentas eventualmente formaron parte de varias combinaciones (discoidales, tubular, globular, esféricas), para crear collares u otro tipo de abalorio corporal. En cuanto, al análisis antropológico o caracterización simbólica, cobra sentido en este caso preguntarse, sí, ¿Los adornos corporales identificados en Nejapa son signos de comportamiento simbólico?, esto quizás constituya una respuesta difícil por resolver sobre todo por el pensamiento simbólico impregnado en quienes elaboraron este tipo de cultura material en el sitio. Cabe destacar, que los adornos corporales junto al cuerpo humano se convierten en elementos de relación con el tipo de prácticas asumidas en sociedades prehispánicas como Nejapa, que de acuerdo al análisis morfológico y simbólico fue eventualmente atado y portado y modificaron la apariencia de quienes la utilizaban y de paso expresaban pleno sentido de comunicación social es decir, "el adorno personal implica la idea de que los objetos decorativos caracteri-
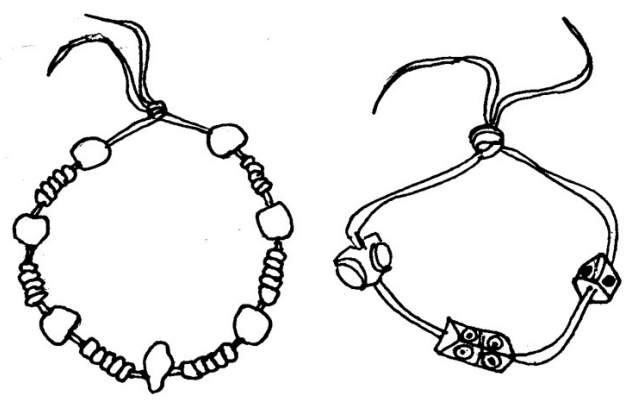

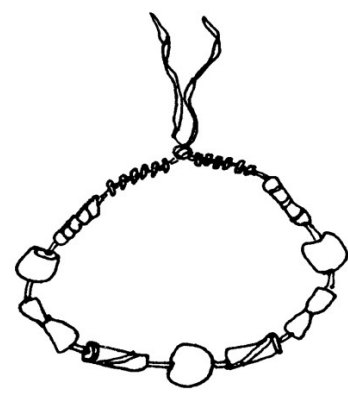

Figura26. Representación hipotética de collar con separadores de diversas formas y tamaño. Cortesía. Dibujo arqueóloga Álvarez, S. común siempre y cuando tuvieran la oportunidad de hacerlo, siendo de esta forma un objeto usado por individuos de ambos sexos y que por naturaleza o apariencia sexual del ser humano (hombre-mujer) se diferenciaban de su semejante. Adicionalmente, sobre este tipo de diferenciación en el uso de adornos corporales tanto entre hombres o mujeres puede sustentarse en la categoría teórica alteridad que "significa aquí un tipo particular de diferenciación. Tiene que ver con la experiencia de lo extraño" (Krotz, 1994:8), en donde la concepción del otro no debe percibirse como individuo sino como alguien que comprehende en conjunto su cultura, además, de formar parte de ella y de expresarla (lo anterior implica un proceso de mediación entre el "yo íntimo" y el "yo social"). Si bien es cierto que precisar la función y significado de los adornos en su conjunto es un riesgo sobre todo por los escasos referentes etnológicos e históricos que permitan determinar estas dos variables formulamos hipótesis interpretativas preliminares que permiten entender que el uso de los adornos analizados se orientaban al hecho de portar collares (hoy llamado prenda) u objetos quizás con un valor protector conocido tradicionalmente como amuleto por lo que el uso de este tipo de adorno corporal es testimonio irrebatible de la cultura material y por medio de éste se puede sin dudas "llegar a conocer el alma humana" (Sarmiento, 2007:221).

De manera, análoga la capacidad simbólica en los adornos se manifiesta con la presencia de formas con características vegetales o geométricas que imitan a un calabazo estilizado o una especie de fruta tropical ácida como la grosella y que en otros casos se hace difícil la identificación botánica de los mis-

zan la apariencia del individuo, ya sea en términos de belleza, prestigio o de cualquier otro tipo" (Renfrew y Bahn, 1993:359).

Sobre esa aseveración no es casualidad entonces que desde las altas o bajas culturas mesoamericanas o sudamericanas este tipo de cultura material históricamente significó una práctica común entre quienes gobernaban (caciques, sacerdotes, guerreros) y gente mos por su marcada estilización. El estudio iconográfico revela implícitamente que estos objetos tienen algún tipo de relación con otras dimensiones de la vida prehispánica y que reflejan la versatilidad del hombre prehispánico de su capacidad de respuesta, adaptación y transformación de su naturaleza mediante su sentido simbólico adquirido a partir, del aprendizaje permanente y continuidad cultural. Ciertamente, en el pensamiento prehispánico el tema de la asociación 


\section{Cuentas con diseños geométricos, frutales y pendiente/colgante
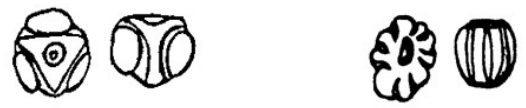

Figuras 27 y 28. Cuentas con diseño geométrico/vegetal posiblemente especie de grosella o calabazo estilizado de $0.6 \mathrm{~mm}$

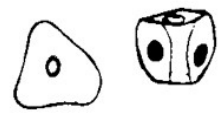

Figuras 29. Cuenta pendiente con diseño geométrico $0.5 \mathrm{~mm}$

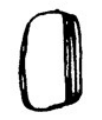

Figuras 30. Colgante sobre piedar de $1.7 \mathrm{~cm} \times 1.3 \mathrm{~cm}$ y de grosor $0.6 \mathrm{~mm}$

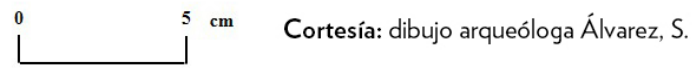

entre ser humano y naturaleza fue frecuente al punto que esa simbiosis quedó representado en su cultura material como sucedió en Nejapa, en donde los objetos funcionaron como mecanismo simbólico.

\section{A modo de consideraciones}

Con esta investigación entregamos al público interesado en la cultura material prehispánica rastros significativos de un sitio arqueológico excepcional, situado en el sur-occidente del municipio de Managua en un área de singular interés configurado geográficamente por la Laguna de Nejapa y el Cerro Motastepe que eventualmente tuvo algún grado de importancia para el aborigen nejapeño. El análisis morfológico permite concluir que para la elaboración de los adornos se utilizaron esencialmente dos materias primas (barro, piedra verde) con la salvedad que hasta ahora en el sitio no se ha determinado algún área fuente de piedras verdes pero la identificación de un ejemplar sugiere hipotéticamente relaciones de intercambio extraregional por lo que el jade fue un elemento esencial en la religión y en la materialización de la cosmovisión de distintas sociedades mesoamericanas (Franca, 2010:327).

A partir, de esas materias primas se identificaron tres tipos de adornos personales elaborados de acuerdo, a patrones estéticos que obedecían a belleza o bien como hoy en día se hace como bisutería personal cotidi- ano, prestigio o como objetos para actividades como el trueque y usados en collares de cuentas, colgantes y extensores usados como narigueras que tenían la función de adorno auricular y que de acuerdo a ciertos teóricos respaldan la idea de que el cuerpo habla por sí solo, funcionando como soporte simbólico. De acuerdo, al análisis antropológico la manufactura de la muestra aportó cierto grado de ornamentación al individuo que vivió en Nejapa aunque, por ahora resulta difícil establecer con seguridad si su empleo se asocia exclusivamente a un grupo determinado lo cierto es que no se puede excluir su uso cotidiano; en su conjunto, una reflexión cierta es que todas las prendas corporales identificados hasta ahora corresponden a una tradición ancestral y catalizadores de los cambios y adaptaciones que diversas sociedades han aplicado a lo largo del tiempo.

En definitiva, este es un estudio preliminar por lo que será necesario incrementar la muestra para poder seguir indagando en torno a este tipo de elementos simbólicos que como hemos señalado es una afirmación de la capacidad de quienes portaban estos objetos situándolos posiblemente en una posición social relativamente importante. 


\section{Referencia bibliográfica}

Balladares, s y Lechado, 1. (2007-2013). Investigaciones Arqueológicas en la Comarca, Nejapa. UNAN-Managua.

Carranza, E. (2008). Análisis Descriptivo de las Cuentas de Collar del Sitio Arqueológico, Nejapa. Ensayo Científico presentado en la XXVII Jornada de Desarrollo Científico, Facultad de Humanidades y Ciencias Jurídicas de UNAN-Managua.

Echeverría, J. (2011). Glosario de Arqueología y Temas a Fines. Tomo I. Quito, Ecuador. Recuperado de https://downloads.arqueo-ecuatoriana.ec/ayhpwxgv/noticias/publicaciones/ INPC-X-GlosarioArqueologiaTomo1.pdf

Echeverría, J. (1981). Glosario Arqueológico. Editorial Gallocapitan. Instituto Otavaleño de Antropología. Otavalo, Ecuador.

Franca, 1. (2010). El Jade y las Piedras Verdes en Teotihuacán, México. Revista do Museu de Arqueología e Etnología de Sao Paulo. 20:327-344. Recuperado de: http://www.periodicos.usp. br/revmae/article/viewFile/89937/92727

Harris, M. (1983). Antropología Cultural. 1era Edición. Alianza Editorial. 622 pp.

Krotz, E. (1994). Alteridad y Pregunta Antropológica. Recuperado de: https://eva.udelar.edu.uy/ pluginfile.php/423372/mod_resource/content/1/Krotz,\%20E\%20\%20Alteridades $\% 20$ 1994\%20 (Cap\%20Alteridad\%20y\%20pregunta\%20antropol\%C3\%B3gica).pdf

Lechado, L. (2012). Propuesta de Plan de Gestión Patrimonial en la Comarca Nejapa, Municipio de Managua. Tesis de maestría para optar al grado master Conservación y Gestión del Patrimonio Cultural para El Desarrollo. UNI, Managua. Recuperado de: https://www.academia. edu/14902345/Arqueolog\%C3\%ADa_Nicaragua._Plan_Gesti\%C3\%B3n_Patrimonial_Nejapa_Managua
Renfrew, c y Bahn, P. (1993). Enterramiento Deliberado de Restos Óseos Humanos. En "Arqueología, Teorías, Métodos y Práctica”. Ed. Akal, España.

Salgado, S y Guerrero, J. (2005). La Distribución de la Jadeíta en Centroamérica y su Significado Social. Cuadernos de Antropología N ${ }^{\circ} 15$, 5354. Recuperado de: https://revistas.ucr.ac.cr/ index.php/antropologia/article/view/10761

Sarmiento, I. (2007). Cultura y Cultura Material: Aproximaciones a los conceptos e Inventario Epistemológico. Anales del Museo de América, \#15:217-236. Recuperado de:http://digibuo. uniovi.es/dspace/bitstream/10651/20599/1/ CulturaYCulturaMaterial.pdf

\section{Agradecimientos}

En principio, a la coordinación del CADI-UNAN-Managua por facilitar la muestra de estudio; así, mismo expresar mi gratitud a Emilio Carranza egresado de la carrera de Historia con Orientación en Arqueología por ser quien realiza un primer acercamiento preliminar de cuentas de collar del sitio en cuestión en el año 2004 en el marco de las Prácticas de Profesionalización II; agradezco también, la valiosa contribución de la arqueóloga Scarleth Álvarez por realizar los dibujos y finalmente, como en otras oportunidades agradecer a aquellos evaluadores y lectores anónimos por sus aportes y revisión del manuscrito final.

\section{Notas}

1 El sitio está fuertemente amenazado por la posible construcción de un proyecto residencial además, de las actividades de siembro.

2 Es el testigo o evidencia que una actividad determinada deja en un sitio o yacimiento arqueológico (ej. Construcción, madrigueras y otros.).

3 Para el análisis morfológico se retoman datos técnicos de glosarios arqueológicos y de imágenes que aparecen en la web sobre terminología de cuentas de collar en varios sitios arqueológicos a nivel global. 


\section{Kevin Ernesto González Hodgson}

Bilwi, Puerto Cabezas, RACCN (1985). Licenciado en Historia con Mención en Arqueología por la UNAN-Managua (2009) y máster en Métodos de Investigación Científica por la Facultad Regional Multidisciplinaria (FAREM)-Carazo, UNAN-Managua con una tesis sobre la "Identidad socio-cultural a partir, de la Producción de diseños cerámicos en San Juan de Oriente, Masaya". Docente-investigador del Centro Arqueológico de Documentación e Investigación (CADI-UNAN), quien ha realizado y participado en diversas investigaciones arqueológicas en la Costa Caribe, Río San Juan, León y Managua y con experiencia en docencia de formación continua. Sus áreas de interés son arqueología histórico-cultural, iconografía y semiótica o bien estudios relacionados a antropología simbólica generados a partir, del cultural material. Finalmente, el autor coordinó el libro "Conociendo Nuestra Historia: Morrito y sus Comunidades" en el marco del proyecto "Un Estudio Local para el Desarrollo: Historia de Río San Juan y sus Municipios”, promovido por el Departamento de Historia. 